\title{
Joint Optimization of Anchor Deployment and Power Allocation in Wireless Network Localization
}

\author{
Zhaohui Ma, Fan Liu, Member, IEEE, Haidong Qiao, Songzhuo Liu, and Xin Lv
}

\begin{abstract}
In this letter, we propose an optimization based method for joint anchor deployment and power allocation for achieving high-accuracy localization in the wireless network, where we aim at minimizing the localization error bound under a given sensor number and a fixed power budget. By exploiting squared position error bound as the localization metric, the problem is formulated into a mixed boolean second order cone programming, and is then solved by binary particle swarm optimization. Finally, the proposed algorithm is validated by numerical simulations.
\end{abstract}

Index Terms-indoor positioning, anchor deployment, power allocation, squared position error bound (SPEB), second-order cone programming (SOCP), binary particle swarm optimization (BPSO).

\section{INTRODUCTION}

W IRELESS network localization (WNL) has been extensively studied for it can provide high-accuracy location information in situation where global navigation satellite system fails to work, especially in the indoor environment. Such information is crucial in context-aware and ambient intelligence applications. In general, the localization process in WNL is to estimate the position of a mobile node (agent) by range measurement with the aid of a collection of fixed nodes (anchors), whose positions are known a priori.

For such network, the localization accuracy depends on both the anchors' distribution and the measurement performance, where power allocation among the anchors plays an important role. To develop deployment strategies for the anchors, the work in [1] focuses on calculating an optimal placement for sensors using geometric dilution of precision (GDOP), which is unable to analyze the impact of the power. In [2], a nearoptimal node deployment algorithm has been proposed by adding auxiliary nodes to improve network performance. Pioneered by [3], an equivalent Fisher information matrix (EFIM) and the associated squared position error bound (SPEB) have

Manuscript received November 27, 2019; revised January 11, 2020; accepted February 5, 2020. The associate editor coordinating the review of this paper and approving it for publication was Prof. Haewoon Nam. This work was supported in part by the European Union's Horizon 2020 research and innovation programme under the Marie Skłodowska-Curie Grant Agreement No. 793345, in part by the National Natural Science Foundation of China under Grant 61901040, in part by the Major Instrument Project of the National Natural Science Foundation of China under Grant 61527805 , and in part by the Joint Research Fund in Astronomy (U1631123) under a cooperative agreement between the National Natural Science Foundation of China and the Chinese Academy of Sciences. (Corresponding author: Fan Liu)

Z. Ma, H. Qiao, S. Liu and X. Lv are with Beijing Key Laboratory of Millimeter Wave and Terahertz Technology, School of Information and Electronics, Beijing Institute of Technology, Beijing 100081, China

F. Liu is with Department of Electronic and Electrical Engineering, University College London, London WC1E 7JE, U.K. (e-mail: fan.liu@ucl.ac.uk) been defined to build a general framework to address the issues of accuracy and power allocation in WNL. Inspired by this, a posterior Cramér-Rao lower bound (PCRLB) has been derived in [4] for the dynamic network. More recently, a number of investigations have been done on power allocation for WNL [5], [6]. To the best of our knowledge, however, little efforts have been taken towards the direction of joint anchor deployment and power allocation except for [7], where a joint scheme of antenna selection and power allocation for localization has been proposed for radar sensor networks.

This letter aims at achieving the optimal positioning performance under a given anchor number and the total power budget for WNL, where we focus on minimizing the SPEB for WNL by optimizing the deployment of anchors and the power allocated to them. The optimization problem is formulated as a mixed boolean second-order cone programming (SOCP), which is non-convex and is then solved by binary particle swarm optimization (BPSO). Finally, we provide numerical results to assess the effectiveness and the performance of the proposed approach.

\section{SYSTEM MODEL AND PERFORMANCE METRIC}

In this section, we detail the system model and derive a performance metric for localization accuracy in WNL.

\section{A. System Model}

Consider a 2-D network with $N_{\mathrm{b}}$ points for deploying anchors and $N_{\text {a }}$ points that can be reached by an agent, which belong to the point sets $\mathcal{N}_{\mathrm{b}}$ and $\mathcal{N}_{\mathrm{a}}$, respectively. Let $\mathbf{p}_{n}^{\mathrm{b}} \in \mathbb{R}^{2}$ and $\mathbf{p}_{m}^{\mathrm{a}} \in \mathbb{R}^{2}$ be the positions of the $n$th and the $m$ th point in $\mathcal{N}_{\mathrm{b}}$ and $\mathcal{N}_{\mathrm{a}}$. Accordingly, the distance and the angle parameters from $\mathbf{p}_{n}^{\mathrm{b}}$ to $\mathbf{p}_{m}^{\mathrm{a}}$ are denoted by $d_{n, m}$ and $\phi_{n, m}$. The power allocation vector $(\mathrm{PAV})$ is denoted as $\mathbf{x}=\left[x_{1}, x_{2}, \ldots, x_{N_{\mathrm{b}}}\right]^{\mathrm{T}}$, with $x_{n}$ being the energy allocated to the $n$th point of $\mathcal{N}_{\mathrm{b}}$. Correspondingly, the anchor configuration vector (ACV) is denoted as $\mathbf{y}=\left[y_{1}, y_{2}, \ldots, y_{N_{\mathrm{b}}}\right]^{\mathrm{T}}$, where $y_{n}$ is set as 1 if the $n$th point is selected to place an anchor and 0 otherwise.

\section{B. Performance Metric of Localization}

Given the PAV $\mathbf{x}$ and the ACV $\mathbf{y}$, the localization accuracy for a point $\mathbf{p}_{m}^{\mathrm{a}}$ can be measured by the SPEB as follows [3]

$$
\mathcal{P}\left(\mathbf{p}_{m}^{\mathrm{a}} ; \mathbf{x}, \mathbf{y}\right) \triangleq \operatorname{tr}\left\{\mathbf{J}_{\mathrm{e}}^{-1}\left(\mathbf{p}_{m}^{\mathrm{a}} ; \mathbf{x}, \mathbf{y}\right)\right\}
$$

where $\operatorname{tr}\{\cdot\}$ is the trace operator, $\mathbf{J}_{\mathrm{e}}\left(\mathbf{p}_{m}^{\mathrm{a}} ; \mathbf{x}, \mathbf{y}\right)$ is the EFIM for $\mathbf{p}_{m}^{\mathrm{a}}$. which can be written as

$$
\mathbf{J}_{\mathrm{e}}\left(\mathbf{p}_{m}^{\mathrm{a}} ; \mathbf{x}, \mathbf{y}\right)=\sum_{n=1}^{N_{\mathrm{b}}} \xi_{n, m} \cdot x_{n} \cdot y_{n} \cdot \mathbf{u}_{n, m} \mathbf{u}_{n, m}^{\mathrm{T}}
$$


where $\mathbf{u}_{n, m}=\left[\cos \left(\phi_{n, m}\right), \sin \left(\phi_{n, m}\right)\right]^{\mathrm{T}}, \xi_{n, m}=\zeta_{n, m} / d_{n, m}^{\beta}$ is the equivalent ranging coefficient (ERC) associated with ranging coefficient (RC) $\zeta_{n, m}>0$ and the amplitude loss exponent (ALE) $\beta \geq 2$ that represents the energy attenuation.

By substituting (2) into (1), the SPEB can be recast as

$$
\mathcal{P}\left(\mathbf{p}_{m}^{\mathrm{a}} ; \mathbf{x}, \mathbf{y}\right)=\frac{4 \cdot \mathbf{1}^{\mathrm{T}} \mathbf{R}_{m}(\mathbf{x} \circ \mathbf{y})}{(\mathbf{x} \circ \mathbf{y})^{\mathrm{T}} \mathbf{R}_{m}^{\mathrm{T}} \boldsymbol{\Lambda}_{m} \mathbf{R}_{m}(\mathbf{x} \circ \mathbf{y})}
$$

where $\circ$ denotes Hadamard product, $\mathbf{R}_{m}=\operatorname{diag}\left\{\xi_{1, m}, \xi_{2, m}, \ldots\right.$, $\left.\xi_{N_{\mathrm{b}, m}}\right\}$ is the ERC matrix, and $\boldsymbol{\Lambda}_{m}$ is an $N_{\mathrm{b}} \times N_{\mathrm{b}}$ matrix, with its $(i, j)$-th entry being $\left[\boldsymbol{\Lambda}_{m}\right]_{i, j}=2 \sin ^{2}\left(\phi_{i, m}-\phi_{j, m}\right)$.

\section{JOINT OPTIMIZATION OF THE ANCHORS' OPTIMAL DEPLOYMENT UNDER OPTIMAL POWER ALLOCATION}

In this section, we formulate an optimization problem aiming for minimizing the SPEB defined in (3) via optimally deploying the anchors and allocating the power. While the problem is non-convex, we propose a BPSO-combined algorithm to obtain a sub-optimal solution.

\section{A. Problem Formulation}

Before the formulation, we will show an equivalent form of SPEB. Let us firstly set an exclusive upper-bound $\eta_{m}$ for (3) as

$$
\frac{4 \cdot \mathbf{1}^{\mathrm{T}} \mathbf{R}_{m}(\mathbf{x} \circ \mathbf{y})}{(\mathbf{x} \circ \mathbf{y})^{\mathrm{T}} \mathbf{R}_{m}^{\mathrm{T}} \boldsymbol{\Lambda}_{m} \mathbf{R}_{m}(\mathbf{x} \circ \mathbf{y})} \leq \eta_{m}, m \in \mathcal{N}_{\mathrm{a}}
$$

Following [6], (4) can be equivalently expressed in the form of a second-order cone (SOC) as

$$
\left\|\mathbf{A}_{m} \mathbf{R}_{m}(\mathbf{x} \circ \mathbf{y})+\mathbf{b}_{m}\right\|_{2} \leq \mathbf{1}^{\mathbf{T}} \mathbf{R}_{m}(\mathbf{x} \circ \mathbf{y})-2 \eta_{m}^{-1}, m \in \mathcal{N}_{\mathrm{a}}
$$

where $\mathbf{A}_{m}=[\mathbf{c}(2 \phi), \mathbf{s}(2 \phi), \mathbf{0}]^{\mathrm{T}}, \mathbf{c}(\phi)=\left[\cos \left(\phi_{m, 1}\right), \cos \left(\phi_{m, 2}\right), \ldots\right.$, $\left.\cos \left(\phi_{m, n}\right)\right]^{\mathrm{T}}, \mathbf{s}(\phi)=\left[\sin \left(\phi_{m, 1}\right), \sin \left(\phi_{m, 1}\right), \ldots, \sin \left(\phi_{m, n}\right)\right]^{\mathrm{T}}$ and $\mathbf{b}_{m}=\left[0,0, \eta_{m}^{-1}\right]^{\mathrm{T}}$.

Let us denote $P_{\text {tot }}$ the power budget, $x_{\max }$ the maximum power that can be allocated to an anchor, and $L$ the number of anchors to be deployed. The optimization problem that minimizes the maximum SPEB of the points from $\mathcal{N}_{\mathrm{a}}$ under given anchor number and power budget can be formulated as

$$
\begin{aligned}
\mathscr{P}: \min _{\mathbf{x}, \mathbf{y}} & \eta \\
\text { s.t. } & \mathbf{1}^{\mathrm{T}} \mathbf{y}=L \\
& \mathbf{1}^{\mathrm{T}} \mathbf{x} \leq P_{\text {tot }} \\
& y_{n} \in\{0,1\}, n \in \mathcal{N}_{\mathrm{b}} \\
& 0 \leq x_{n} \leq x_{\max }, n \in \mathcal{S} \subset \mathcal{N}_{\mathrm{b}} \\
& x_{n}=0, n \in \mathcal{N}_{\mathrm{b}} \backslash \mathcal{S} \\
& \left\|\mathbf{A}_{m} \mathbf{R}_{m}(\mathbf{x} \circ \mathbf{y})+\mathbf{b}\right\|_{2} \\
& \leq \mathbf{1}^{\mathbf{T}} \mathbf{R}_{m}(\mathbf{x} \circ \mathbf{y})-2 \eta^{-1}, \forall m \in \mathcal{N}_{\mathrm{a}}
\end{aligned}
$$

where $\mathbf{b}=\left[0,0, \eta^{-1}\right]^{\mathrm{T}}, \mathcal{S}$ is the subset of $\mathcal{N}_{\mathrm{b}}$ that contains the points being deployed an anchor. (6) is non-convex since the third constraint of (6) restricts $\mathbf{y}$ to be a boolean vector, despite the fact that the objective as well as the other constraints are convex. Consequently, (6) is difficult to be solved straightforwardly.

\section{B. BPSO-Combined Optimization}

To tackle this issue, we propose an algorithm based on BPSO [8], which is modified from PSO [9] to solve boolean optimization problem. In the algorithm, $\mathbf{y}$ is iteratively updated by BPSO under the constraint $\mathbf{1}^{\mathrm{T}} \mathbf{y}=L$. By doing so, (6) can be converted to an SOCP that yields an optimal $\mathbf{x}$ given $\mathbf{y}$. In the following we will detail the algorithm.

Given $N_{\mathrm{p}}$ particles that belong to the set $\mathcal{N}_{\mathrm{p}}$, the maximum iteration time $N_{\mathrm{i}}$ and let the position of each particle be an $\mathrm{ACV}$, the position of the $i$ th particle after $j$ th iteration is denoted as

$$
\mathbf{y}_{i}^{j}=\left[y_{i, 1}^{j}, y_{i, 2}^{j}, \ldots, y_{i, N_{\mathrm{b}}}^{j}\right]^{\mathrm{T}}, y_{i, n}^{j} \in\{0,1\}, \mathbf{1}^{\mathrm{T}} \mathbf{y}=L
$$

The velocity of the $i$ th particle after $j$ th iteration is denoted as $\mathbf{v}_{i}^{j} \in \mathbb{R}^{N_{\mathrm{b}}}$, whose $n$th entry can be expressed as

$$
v_{i, n}^{j}=\omega v_{i, n}^{j-1}+c_{1} r_{1}\left(p_{i, n}-y_{i, n}^{j-1}\right)+c_{2} r_{2}\left(g_{n}-y_{i, n}^{j-1}\right)
$$

where $p_{i, n}$ is the $n$th entry of the $i$ th particle's personal best position $\mathbf{p}_{i}$, and $g_{n}$ is the $n$th entry of the entire swarm's global best position g. $r_{1}$ and $r_{2}$ updated before every iteration are random numbers that are uniformly distributed in $[0,1] . \omega$ is the inertia weight, $c_{1}$ and $c_{2}$ are the accelerated factors. Following [8], $\omega$ varies from 0.9 at the beginning of the iteration to 0.4 at the end, $c_{1}$ and $c_{2}$ are set as 2 for balancing the convergence rate and the search ability. Besides, the scale of $v_{i, n}^{j}$ is constrained by $\left[-v_{\max }, v_{\max }\right]$ to avoid data flooding, with $v_{\max }$ being set as 3 .

In every iteration of BPSO, $v_{i, n}^{j}$ is responsible for the value of $y_{i, n}^{j}$ by following the rule that the larger the $v_{i, n}^{j}$, the higher the probability that $y_{i, n}^{j}$ takes a value of 1 . Under the constraint of $\mathbf{1}^{\mathrm{T}} \mathbf{y}=L, y_{i, n}^{j}$ is updated by

$$
y_{i, n}^{j}=\left\{\begin{array}{cc}
1, & v_{i, n}^{j} \geq \sigma_{L}\left(\mathbf{v}_{i}^{j}\right) \\
0, & v_{i, n}^{j}<\sigma_{L}\left(\mathbf{v}_{i}^{j}\right)
\end{array}\right.
$$

where $\sigma_{L}\left(\mathbf{v}_{i}^{j}\right)$ is the $L$ th largest entry of $\mathbf{v}_{i}^{j}$.

As mentioned above, every updated $\mathbf{y}_{i}^{j}$ denotes a configuration of an $L$-anchor deployment. By substituting $\mathbf{y}_{i}^{j}$ into (6), we have an optimization problem as

$$
\begin{aligned}
\mathscr{P}_{\mathrm{s}}: \min _{\mathbf{x}_{i}^{j}} & \eta \\
\text { s.t. } & \mathbf{1}^{\mathrm{T}} \mathbf{x}_{i}^{j} \leq P_{\mathrm{tot}} \\
& 0 \leq x_{i, n}^{j} \leq x_{\max }, n \in \mathcal{S} \subset \mathcal{N}_{\mathrm{b}} \\
& x_{i, n}^{j}=0, n \in \mathcal{N}_{\mathrm{b}} \backslash \mathcal{S} \\
& \left\|\mathbf{A}_{m} \mathbf{R}_{m}\left(\mathbf{x}_{i}^{j} \circ \mathbf{y}_{i}^{j}\right)+\mathbf{b}\right\|_{2} \\
& \quad \leq \mathbf{1}^{\mathbf{T}} \mathbf{R}_{m}\left(\mathbf{x}_{i}^{j} \circ \mathbf{y}_{i}^{j}\right)-2 \eta^{-1}, \forall m \in \mathcal{N}_{\mathrm{a}}
\end{aligned}
$$

$\mathscr{P}_{\mathrm{s}}$ can be solved by standard numerical tool such as CPLEX [10], through which an optimal PAV $\mathbf{x}_{i}^{j}$ corresponds to $\mathbf{y}_{i}^{j}$ is achieved. After that, $\mathcal{P}\left(\mathbf{p}_{m}^{\mathrm{a}} ; \mathbf{x}_{i}^{j}, \mathbf{y}_{i}^{j}\right), \forall m \in \mathcal{N}_{\mathrm{a}}$ are calculated by (3), with either the maximum or the mean of them being set as fitness value $\delta$. The two criteria seem to be intuitively equivalent but they do make some differences on the results, which will be shown in the following numerical simulation. At last, $\delta$ is compared with particle's personal best 
fitness value $f p_{i}$ and swarm's global best fitness value $f g$. If $\delta$ is smaller, $f p_{i}$ and $\mathbf{p}_{i}$ or $f g$ and $\mathbf{g}$ are updated by $\delta$ and $\mathbf{y}_{i}^{j}$.

Remark 1: It should be highlighted that the SOCP in (10) is a subproblem of BPSO, which is solved for each particle per iteration. Accordingly, the fitness value $\delta$ calculated for $\mathbf{x}$ and $\mathbf{y}$ can be seen as a performance evaluation of the obtained results. By designing the evaluation criterion in different strategies such as minimizing the maximum SPEB or minimizing the mean of SPEB in each iteration, we set a global guidance for the BPSO to search for the preferable result.

The proposed algorithm for joint optimization of anchor deployment and power allocation in WNL is thus given as Algorithm 1.

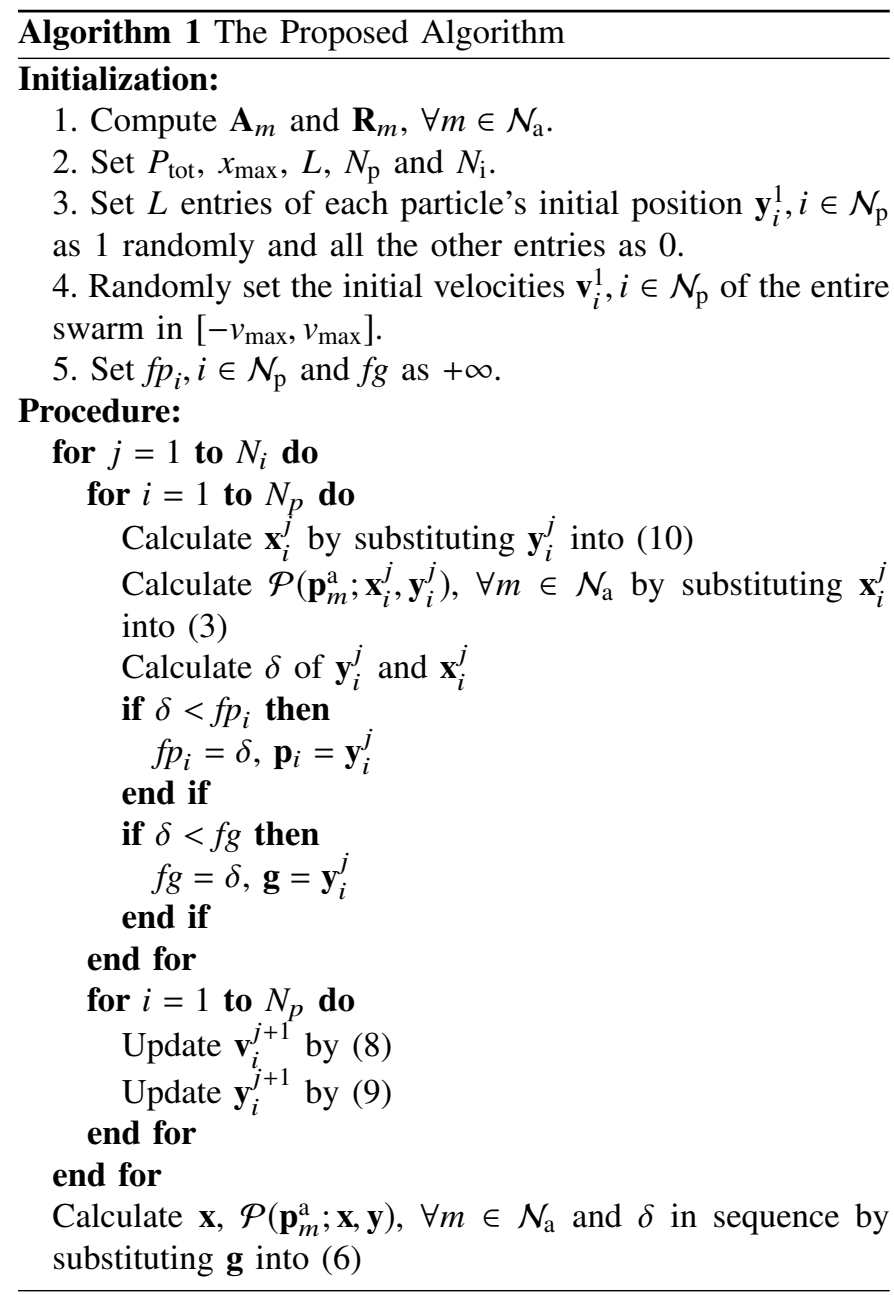

\section{NUMERICAL SIMULATION AND RESULTS}

In this section, Monte-Carlo simulations are executed on 2-D scenes to verify the algorithm's performance. By using the proposed algorithm, the performance of anchors' optimal deployment (OD) with min-max and min-mean SPEB under optimal power allocation (OPA) are compared with that of strategies including random deployment (RD) under uniform power allocation (UPA) and OD in [7] under OPA. To further clarify the meaning of OD in [7], we summarize it as a twostep optimization. Firstly, $\forall n \in \mathcal{N}_{\mathrm{b}}$ are taken into consideration

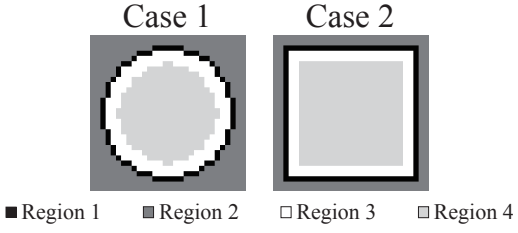

Fig. 1. Two exemplary scenes in pixel-form

Region 1: Points that non-transparent to radio but can hold anchors beside Region 2: Points that non-transparent to radio and can't hold an anchor Region 3: Points that transparent to radio (air) and an agent can't reach Region 4: Points that transparent to radio (air) and an agent can reach

in OPA as similar to (6). Secondly, the points that are allocated $L$ largest powers are selected as the anchors' OD, and OPA is run again for the selected points. Here we note that by RD, we mean randomly choosing $L$ points from $\mathcal{N}_{\mathrm{b}}$ to deploy anchors for multiple times, from which we expect a strategy without any optimization.

We consider two basic topologies as Case 1 and 2 in Fig. 1 , both of which are $[20 m \times 20 m] 2$-D scenes. Each scene is split into 4 regions as illustrated below Fig. 1. Our aim is to place the anchors on the points belong to region 1 such that the agents can reach any point in region 4 . The choice of a reasonable $L$ can be referred to [11]. Here we run the simulation with $L=3$ and $L=4$ for simplicity. The population of particle swarm $N_{\mathrm{p}}$ is usually set comparable to the dimension of a particle and we set it as 50 in the simulation. $N_{\mathrm{i}}$ is set as 30 . Without loss of generality, we assume that the $\mathrm{RC} \zeta_{n, m}=1000, \forall n \in \mathcal{N}_{\mathrm{b}}, m \in \mathcal{N}_{\mathrm{a}}{ }^{1}$, the ALE $\beta=2$, the total power $P_{\text {tot }}$ is $1 W$, and finally $x_{\max }$ is $50 \%$ of $P_{\text {tot }}$. All the optimization problems are solved by CPLEX [10].

The anchor deployment results are shown in Fig. 2. It can be observed that for min-max OD the proposed algorithm always tends to uniformly deploy the anchors. For min-mean OD, the proposed algorithm may counter-intuitively deploy anchors on one half of the scene except for Case 2 with $L=4$. As for the algorithm in [7], its deployment strategy seems to fall between the aforementioned two counterparts but shows similar results to that of min-max OD and min-mean OD in Case 2. The corresponding power allocation results in percentage of $P_{\text {tot }}$ are listed as Table I, where we note that UPA is not always the best choice unless for some symmetric deployments of anchors.

Comparisons of localization accuracy between different algorithms are given in terms of SPEB. It is worth highlighting that when we execute the optimization algorithm, RC $\zeta_{n, m}$ is set as 1000 to keep the iteration consistently effective. Then when we calculate the SPEB as the final results to present, the RCs are set both as constant 1000 to represent the ideal condition and Rayleigh distributed variables with mean 1000 to support the evaluation in the practical condition. ${ }^{2}$ These two kinds of results are illustrated in Fig. 3 and Fig. 4. The results are given in the form of the $\max$, the $\min$ and the

\footnotetext{
${ }^{1}$ The derivation of RCs can be resorted to [3].

${ }^{2}$ We here set the RCs as Rayleigh distributed numbers to represent the signal's fading model. In fact, similar models can be achieved by other distributions for RCs.
} 


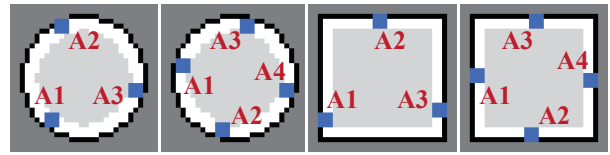

(a)

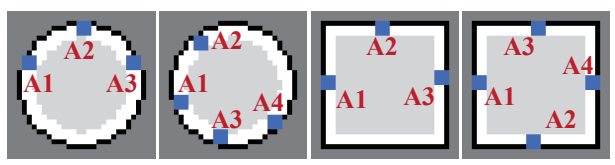

(b)

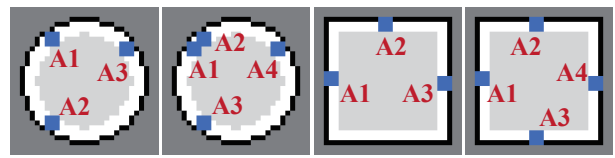

(c)

Fig. 2. The anchor deployment results of: (a) min-max OD, (b) min-mean $\mathrm{OD}$ and (c) OD in [7].

TABLE I

OPA Results for Corresponding OD in Percentages of $\boldsymbol{P}_{\text {tot }}$

\begin{tabular}{|c|c|c|c|c|}
\hline & Anchor & min-max OD & min-mean OD & OD in [7] \\
\hline \multirow{3}{*}{$\begin{array}{c}\text { Case } 1 \\
L=3\end{array}$} & A1 & $40.53 \%$ & $30.19 \%$ & $34.04 \%$ \\
\hline & A2 & $29.60 \%$ & $26.42 \%$ & $31.32 \%$ \\
\hline & A3 & $29.87 \%$ & $43.39 \%$ & $34.64 \%$ \\
\hline \multirow{4}{*}{$\begin{array}{c}\text { Case } 1 \\
L=4\end{array}$} & A1 & $25.00 \%$ & $39.47 \%$ & $12.67 \%$ \\
\hline & A2 & $25.00 \%$ & $14.09 \%$ & $12.67 \%$ \\
\hline & A3 & $25.00 \%$ & $18.96 \%$ & $37.33 \%$ \\
\hline & A4 & $25.00 \%$ & $27.47 \%$ & $37.33 \%$ \\
\hline \multirow{3}{*}{$\begin{array}{c}\text { Case } 2 \\
L=3\end{array}$} & A1 & $27.65 \%$ & $38.67 \%$ & $31.42 \%$ \\
\hline & A2 & $39.87 \%$ & $31.47 \%$ & $41.36 \%$ \\
\hline & A3 & $32.48 \%$ & $29.85 \%$ & $27.22 \%$ \\
\hline \multirow{4}{*}{$\begin{array}{c}\text { Case } 2 \\
L=4\end{array}$} & A1 & $25.00 \%$ & $24.77 \%$ & $25.00 \%$ \\
\hline & $\mathrm{A} 2$ & $25.00 \%$ & $25.38 \%$ & $25.00 \%$ \\
\hline & A3 & $25.00 \%$ & $24.59 \%$ & $25.00 \%$ \\
\hline & A4 & $25.00 \%$ & $25.26 \%$ & $25.00 \%$ \\
\hline
\end{tabular}

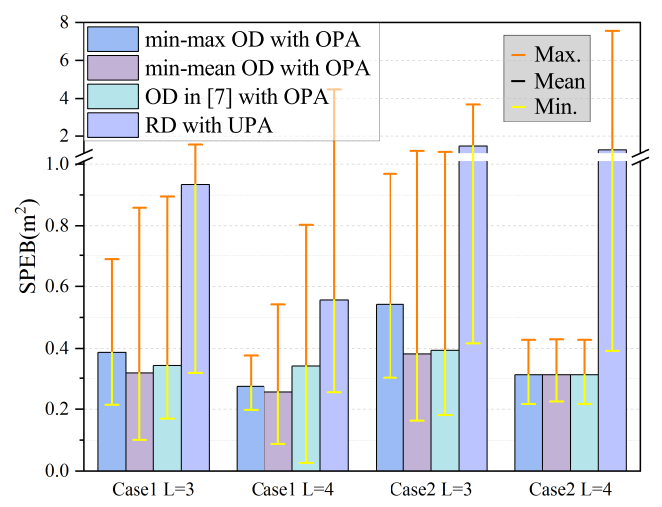

Fig. 3. Comparison of localization accuracy between different algorithms in terms of the SPEB when RC is set as constant.

mean of SPEB, respectively. It is not surprising to see that the localization accuracy becomes worse under the practical condition. Furthermore, while deployments with $L=3$ indeed yield smaller number of anchors required than that of $L=4$, the price is higher SPEB. Then it is noteworthy that in most cases the two proposed ODs under OPA schemes can significantly reduce SPEB, compared to the OD in [7] under OPA and RD under UPA in both the ideal and the practical conditions, which proves the effectiveness of the proposed

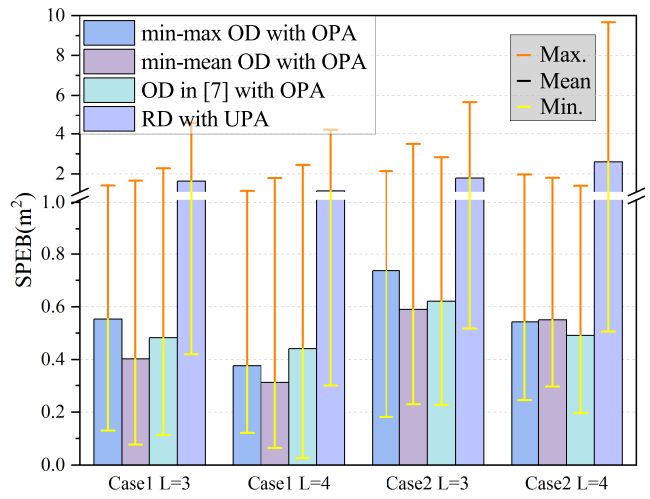

Fig. 4. Comparison of localization accuracy between different algorithms in terms of the SPEB when RC is set as Rayleigh distribution.

algorithm and its controllability on the results. It can be observed from all the results above that a lower mean SPEB usually comes with a higher max SPEB. Exception is Case 2 with $L=4$ in which both the min-max and min-mean OD under OPA yield similar results. This reveals that there exists a trend that the two OD strategies become equivalent under special constraints.

\section{CONCLUSION}

In this letter, we have proposed an optimization approach for solving the joint anchor deployment and power allocation problem in WNL. By employing SPEB as the localization metric, the problem has been formulated into a mixed boolean SOCP problem and then solved by the BPSO algorithm, in which SOCP was utilized for fitness value calculation. Numerical results have been provided to validate the effectiveness of the proposed approach, which show that the OD under OPA could offer a considerably better SPEB performance than that of conventional benchmark methods.

\section{REFERENCES}

[1] N. Kirchhof, "Optimal placement of multiple sensors for localization applications," in Proc. IPIN'2013, 2013, pp. 1-10.

[2] Z. Liu, W. Dai, and M. Z. Win, "Node placement for localization networks,' in Proc. ICC'2017, 2017, pp. 1-6.

[3] Y. Shen and M. Z. Win, "Fundamental limits of wideband localization-part i: A general framework," IEEE Trans. Inf. Theory, vol. 56, no. 10 , pp. 4956-4980, 2010.

[4] S. Li, J. Lv, and S. Tian, "Posterior cramer-rao lower bound for wireless sensor localisation networks," Electron. Lett., vol. 54, no. 22, pp. 12961298, 2018.

[5] W. Dai, Y. Shen, and M. Z. Win, "A computational geometry framework for efficient network localization," IEEE Trans. Inf. Theory, vol. 64, no. 2, pp. 1317-1339, 2017.

[6] Y. Shen, W. Dai, and M. Z. Win, "Power optimization for network localization," IEEE/ACM Trans. Netw., vol. 22, no. 4, pp. 1337-1350, 2013.

[7] B. Ma, H. Chen, B. Sun, and H. Xiao, "A joint scheme of antenna selection and power allocation for localization in mimo radar sensor networks," IEEE Commun. Lett., vol. 18, no. 12, pp. 2225-2228, 2014.

[8] J. Kennedy and R. C. Eberhart, "A discrete binary version of the particle swarm algorithm,” in Proc. SMC'1997, vol. 5, 1997, pp. 4104-4108.

[9] Y. Shi et al., "Particle swarm optimization: developments, applications and resources," in Proc. CEC'2001, vol. 1, 2001, pp. 81-86.

[10] IBM. (2019) 'CPLEX Optimizer'. [Online]. Available: https://www.ibm.com/analytics/cplex-optimizer

[11] W. Dai, Y. Shen, and M. Z. Win, "On the minimum number of active anchors for optimal localization,” in Proc. GLOBECOM'2012, 2012, pp. 4951-4956. 